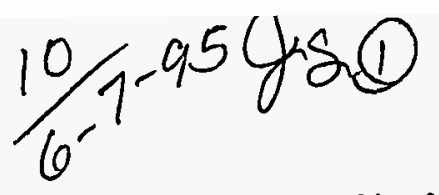

\title{
Injection Envelope Matching in Storage Rings ${ }^{\dagger}$
}

\author{
M. G. Minty and W. L. Spence \\ Stanford Linear Accelerator Center, Stanford University, Stanford, CA 94309 USA
}

\begin{abstract}
The-shape and size of the transverse phase space injected into a storage ring can be deduced from turn-by-turn measurements of the transient behavior of the beam envelope in the ring. Envelope oscillations at $2 \times$ the $\beta$-tron frequency indicate the presence of a $\beta$-mismatch, while envelope oscillations at the $\beta$-tron frequency are the signature of a dispersion function mismatch. Experiments in injection optimization using synchrotron radiation imaging of the beam and a fast-gated camera at the SLC damping rings are reported.

\section{Introduction}

The repetition rate requirements of a linear collider allow the beams to be damped for only a few $\beta$-tron amplitude damping times-about 2.3 for the SLC electron beam at $120 \mathrm{~Hz}$. Since the tolerance on the extracted beam emittances is also stringent, the maximum acceptable injected emittance is similarly restricted. It must include effective increases in the injected beam emittance taking place in the ring itself, on time scales short compared to the radiation damping time. Envelope 'mismatches' are a potentially serious source of such emittance growth.
\end{abstract}

\section{Transverse Dynamics}

A (stably) stored beam envelope will usually asymptotically approach a stationary form, in which its variation around the ring is mainly determined by the magnetic focusing lattice. The stationary envelope is the unique envelope that has the ring's periodicity, and so does not vary from turn-to-turn at any fixed location. Although the shape of the envelope is asymptotically independent of the envelope with which one injects, its size or phase space volume may not be. The time scales for damping and stochastic excitation, which produce initial condition independent equilibria, are typically much longer than those that establish the stationary envelope.

An arbitrary injected beam will not have an envelope shape that 'matches' the symmetry of the ring, and will consequently undergo coherent oscillations, ' $\beta$-beats', at twice the incoherent $\beta$-tron frequency. If the phase advancé varies with respect to incoherent $\beta$-tron amplitude or energy (due to geometric and chromatic non-linearities in the lattice), and the beam contains a spread with respect to these dynamical variables, the $\beta$-beat will decohere (Landau 'damp') and the phase space volume will filament. The Liouville 'invariant' emittance $\epsilon=\sqrt{\left\langle x^{2}\right\rangle\left\langle x^{\prime 2}\right\rangle-\left\langle x x^{\prime}\right\rangle^{2}}$ increases. The matched-equivalent emittance

$$
\bar{\epsilon}=\frac{\left\langle x^{2}\right\rangle+\left\langle\left(\beta x^{\prime}+\alpha x\right)^{2}\right\rangle}{2 \beta}=B \epsilon \geq \epsilon
$$

where $\beta(s)$ is the unique (ring-turn) periodic, Iattice determined, 'matched' envelope shape function $\left(\alpha=-\beta^{\prime} / 2\right)$. $\bar{\epsilon}$ is the beam average Courant-Snyder invariant, and since it is phase advance invariant, $\bar{\epsilon}$ is conserved in the presence of transport in which there is phase advance spread. If the $\beta$-beat decoherence is complete $\epsilon=\bar{\epsilon}=B_{0} \epsilon_{0}$, the initial matched equivalent emittance. $B$ is thus the multiplicative factor by which the emittance grows, and $B=1$ characterizes the 'matched' case.

In a dispersion function mismatch the off-energy components of the (on-average-energy) injected beam's transverse trajectories are not closed orbits in the ring. The beam transverse coordinate-energy correlation coefficients differ from the unique ring-periodic 'matched' off-energy function $\eta(s)$. The anomaly

$$
\Delta \eta=\eta-\frac{\langle x \delta\rangle}{\left\langle\delta^{2}\right\rangle}
$$

is independent of energy spread in first order, but may contain higher orders. It behaves like a free $\beta$-tron oscillation and so has a dispersion-anomaly invariant $\|\Delta \eta\| / \sqrt{\beta}$, where

$$
\|\Delta \eta\|=\left\{(\Delta \eta)^{2}+\left[\beta\left(\Delta \eta^{\prime}\right)+\alpha(\Delta \eta)\right]^{2}\right\}^{1 / 2}
$$

The matched-equivalent emittance is increased according to $\bar{\epsilon} \rightarrow \bar{\epsilon}+\frac{1}{2}\|\Delta \eta\|^{2}\left\langle\delta^{2}\right\rangle / \beta$, with $\left\langle\delta^{2}\right\rangle$ the injected mean square energy deviation. The effects of anomalous dispersion are damped at the transverse damping rate, and cannot induce synchro- $\beta$-tron coupling.

Observation of the evolution of the beam size over several hundred consecutive turns (a time scale short compared to the filamentation time) at a location of nonvanishing dispersion allows a precise and complete diagnostic of the injection envelope. The mean square spot size at a fixed location with $\beta$-function $\beta$ in a ring with a linear lattice and tune $\nu$, will vary with turn number $n$ according to ${ }^{1}$

$$
\begin{aligned}
\frac{\left\langle x^{2}\right\rangle}{\beta}=B \epsilon+\sqrt{B^{2}-1} \epsilon \cos \left[2 \pi\left(2 n \nu+\mu_{\beta}\right)\right] \\
+2 \eta \frac{\|\Delta \eta\|}{\beta}\left\langle\delta^{2}\right\rangle \cos \left[2 \pi\left(n \nu+\mu_{\eta}\right)\right]
\end{aligned}
$$

for an arbitrary $\beta, \eta$ mismatch.

\section{Instrumentation ${ }^{2}$}

A fast-gated camera manufactured by Xybion Electronic Systems Corporation ${ }^{3}$ detects the visible synchrotron radiation from $1.19 \mathrm{GeV}$ bunches of $\sim 0.5-3.5 \cdot 10^{10} e^{-}$or $e^{+}$ in a $2.0 \mathrm{~m}$ radius of curvature bending field. The camera's fast gating capabilities allow the imaging of a single bunch on a single ring revolution. Since the system bandwidth limits the data acquisition time, images corresponding to 


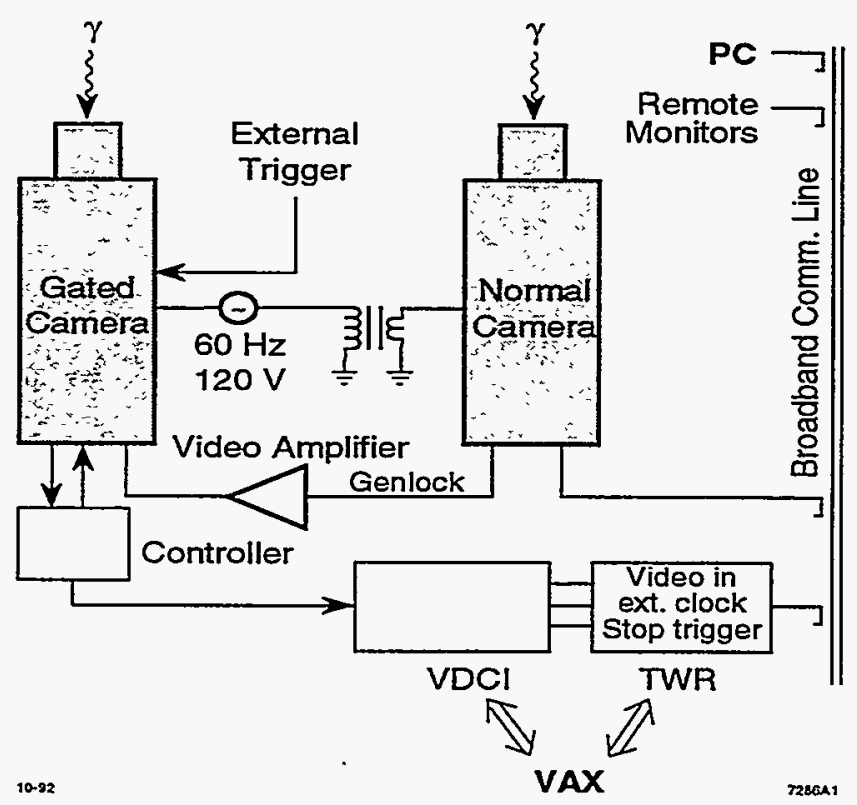

FIG. 1. Data acquisition system.

consecutive turns are actually obtained from different machine pulses. Fig. 1 shows a block diagram of the detection apparatus. A line-locked (normal) camera is used to supply composite synchronization for the gated camera. The signals are read out using a SLAC-built video digitizer clock interface (VDCI), and a $30 \mathrm{MHz} 8 \mathrm{k} \times 8$ bit transient waveform recorder (TWR) - and processed by the SLC control system's VAX using its automated emittance measurement system. ${ }^{4}$ Fig. 2 shows digitized data for the first 12 turns. The data are processed by first digitizing a background image, which is then subtracted from a digitized beam image. The $x$ and $y$-axis projections of the background-subtracted image are found and fit to Gaussians, as shown in Fig. 3.

\section{Diagnostic and Tuning Experiments}

Plotted in Fig. 4 are the measured mean square beam sizes in the $x$ and $y$ planes acquired over the first 100 turns, and their Fourier transforms. The peaks at the betatron frequency and at its second harmonic indicate the presence and size of dispersion and $\beta$-mismatches, respectively, in the injected beam. The phase of the mismatch is also obtained by the Fourier transform. The $\nu$ values obtained from the $2 \nu$ and $\nu$ peaks here, agree with the $\nu$ peak in the beam centroid data. If $\rho$ is the ratio of the strengths of the $\mu=2 \nu$ to the $\mu=0$ peaks, the mismatch measure is $B=1 / \sqrt{1-\rho^{2}}$ according to eq. (4). The equivalent emittance $\bar{\epsilon}$ is just the $\mu=0$ peak, from which the emittance $\epsilon$ follows as $\bar{\epsilon} / B$, which is also the in-quadrature sum of the two peaks. For pure $2 \nu$ oscillations the fractional mean square size variations $\left(\left\langle x^{2}\right\rangle^{\max }-\left\langle x^{2}\right\rangle^{\min }\right) /\left(\left\langle x^{2}\right\rangle^{\max }+\left\langle x^{2}\right\rangle^{\min }\right)=\rho$ as well.

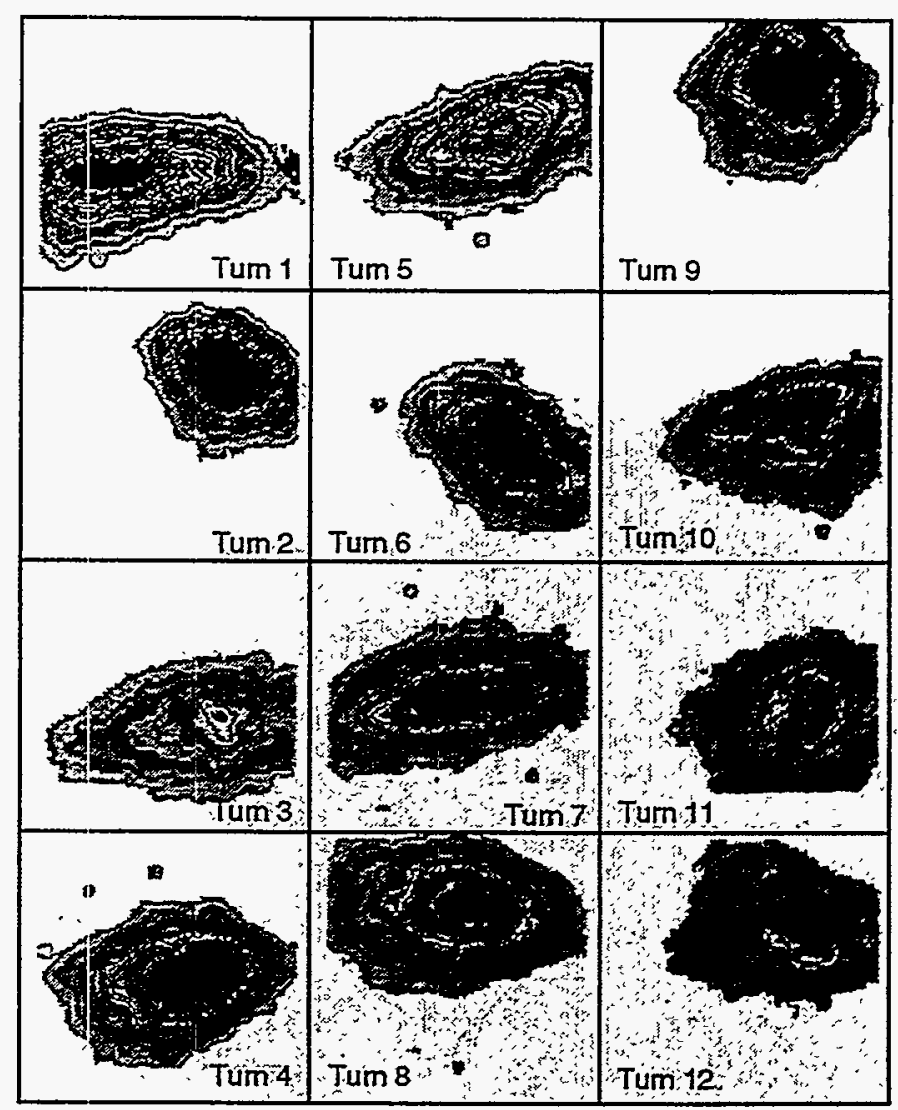

FIG. 2. $x-y$ plane beam images for the first 12 turns in the SLC $e^{-}$damping ring for $\nu_{x} \cong \nu_{y} \approx .25$.

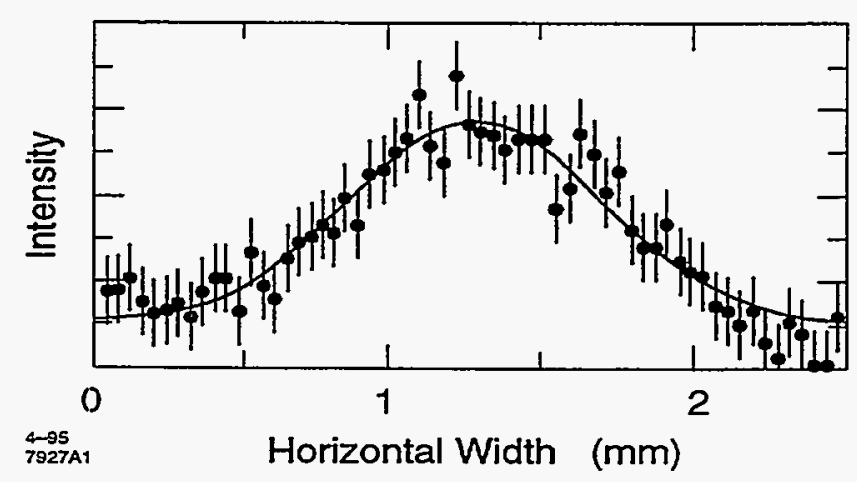

FIG. 3. Plane projected data and Gaussian fit.

Even for quite prominent appearing envelope oscillations $B \sim 1+\frac{1}{2} \rho^{2}$, corresponding to $B$ values not much bigger than $1 . B$ as determined by this technique is free of any dependence on the length scale calibration of the camera. The absolute emittance depends quadratically on this scale, and on the matched $\beta$-function at the observation point-but is otherwise independent of the lattice model. 


\section{DISCLAIMER}

Portions of this document may be illegible in electronic image products. Images are produced from the best available original document. 

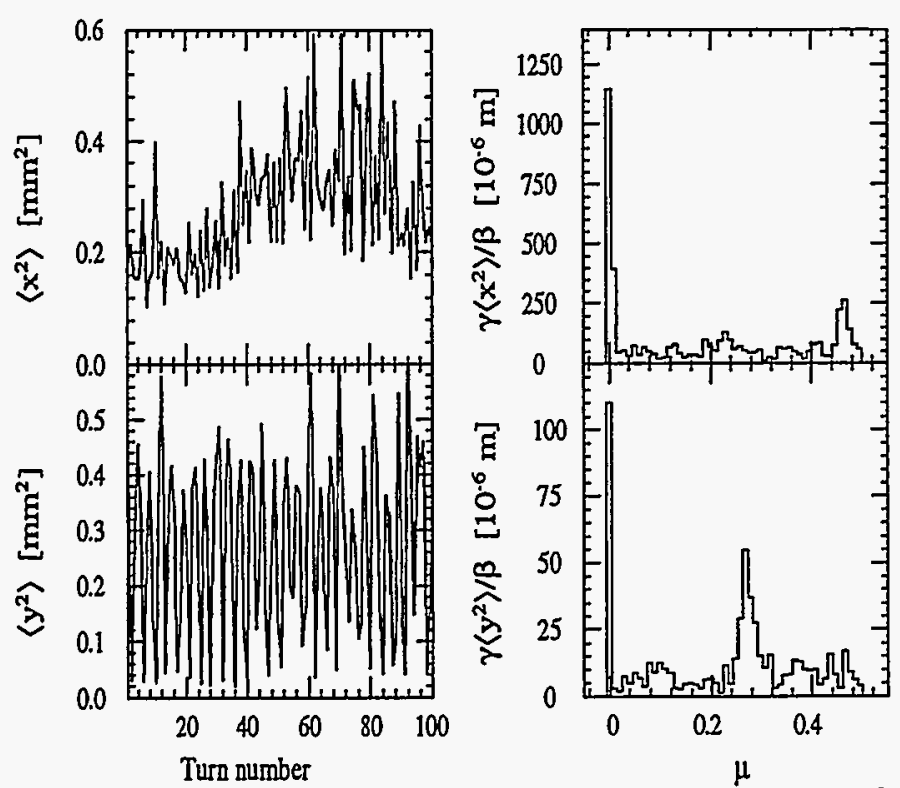

FIG. 4. Mean square beam size turn-by-turn data, and its Fourier transform, normalized to have units of Lorentz invariant emittance. Peaks are evident at $\nu_{x}$ and $2 \nu_{x}$ for $\nu_{x}=.235$, and $1-2 \nu_{y}$ for $\nu_{y}=.365$, indicating a horizontal dispersion function mismatch and $\beta$-mismatches.

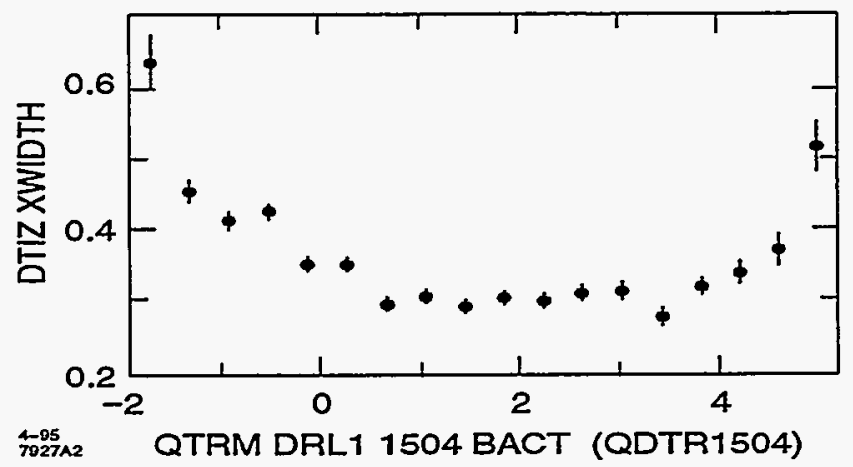

FIG. 5. Filamented rms beam size measured as a function of quadrupole strength.

Optimizing the Match: Gross mismatches can be seen by comparing the beam images corresponding to different turns directly on a tv monitor. The matching quadrupoles in the ring injection line can be used to reduce the differences in the turn-to-turn beam size changes in both the $x$ and $y$ planes. A good match was achieved by measuring the beam sizes after the beam had nearly completely filamented as a function of the strength of the matching quadrupoles. Finding the optimum sampling time involved making a coarse scan in which the trigger time was moved in steps of many hundred turns. Plotted in Fig. 5 is the measured size of the filamented beam at about $0.15 \mathrm{~ms}$ after injection as a function of quadrupole strength. The minimum width corresponds to the optimum match. Ad-
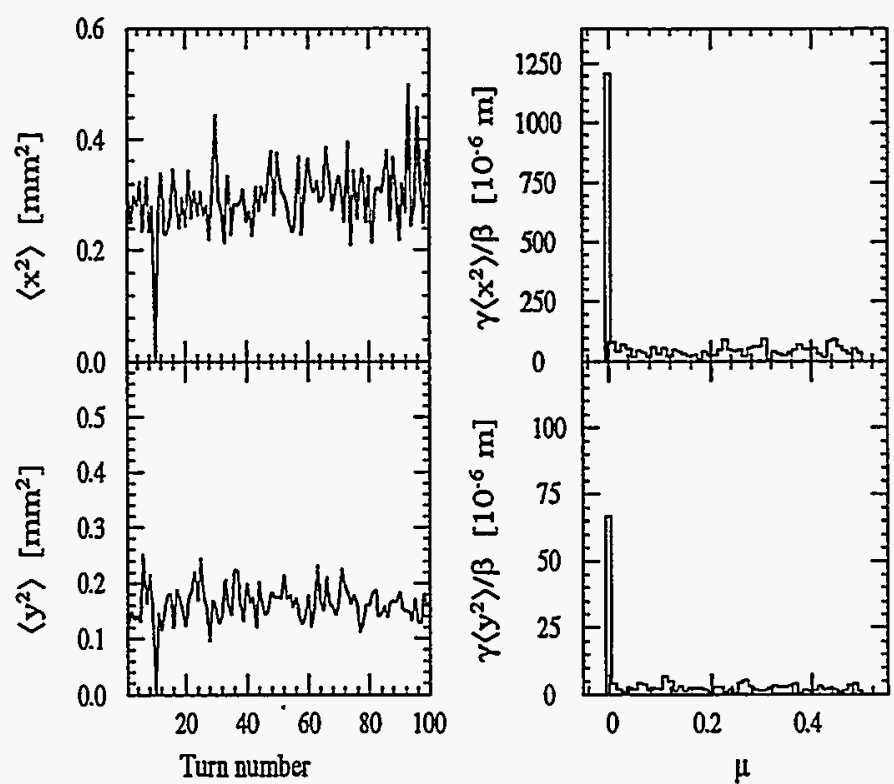

FIG. 6. The same measurements as Fig. 4, after optimizing the match.

justments to this and other quadrupoles yielded a near perfect match as confirmed by the measurements of the first 100 turns shown in Fig. 6.

\section{Prospects}

The methods developed here have also been used to study the effects of coherent centroid motion on the beam envelope. They could easily be extended to include the $x-y$ correlations in the injected beam as long as the ring is operated off all coupling resonances, and to higher moments $\left\langle x^{3}\right\rangle,\left\langle x^{4}\right\rangle$, etc.. Simultaneous sampling from more that one location in the ring can be used to separately determine the energy spread and the injected dispersion function. The observation of higher betatron harmonics would indicate the presence of nonlinearities in the lattice of the ring and could provide a measure of their strength. Given a suitable signal, alternative to synchrotron light, these techniques can be used in hadron machines.

† Work supported by Department of Energy Contract DE-AC03-76SF00515.

1 All orders in $\Delta \eta$ and $\eta$ require the substitutions: $B \epsilon \rightarrow B \epsilon+\left(\eta^{2}+\frac{1}{2}\|\Delta \eta\|^{2}\left\langle\delta^{2}\right\rangle\right) / \beta$ and $\sqrt{B^{2}-1} \epsilon \rightarrow$ $\sqrt{\left(B^{2}-1\right) \epsilon^{2}+\frac{1}{4}\left(\|\Delta \eta\|^{2}\left\langle\delta^{2}\right\rangle / \beta\right)^{2}}$.

${ }^{2}$ M. G. Minty et al. AIP Conf. Proc. 281 (1992) 158.

3 Xybion Corporation, San Diego, CA 92126.

${ }^{4}$ M. C. Ross et al., 1987 IEEE Part. Acc. Conf., 725.

${ }^{5}$ M. G. Minty, A. W. Chao, W. L. Spence, these proceedings.

${ }^{6}$ N. Merminga, P. L. Morton, J. T. Seeman, W. L. Spence, Proc. 1991 IEEE Part. Acc. Conf. (Chicago), 461. 


\section{DISCLAIMER}

This report was prepared as an account of work sponsored by an agency of the United States Government. Neither the United States Government nor any agency thereof, nor any of their employees, makes any warranty, express or implied, or assumes any legai liability or responsibility for the accuracy, completeness, or usefulness of any information, apparatus, product, or process disclosed, or represents that its use would not infringe privately owned rights. Reference herein to any specific commercial product, process, or service by trade name, trademark, manufacturer, or otherwise does not necessarily constitute or imply its endorsement, recommendation, or favoring by the United States Government or any agency thereof. The views and opinions of authors expressed herein do not necessarily state or reflect those of the United States Government or any agency thereof. 